\title{
Comparison of Routing Convention in MANET: A Survey
}

\author{
S. Naga Mallik Raj ${ }^{1}$ and S. Neeraja ${ }^{2}$ \\ ${ }^{1}$ Dept. of Department of Computer Science and Engineering, Vignan's Institute of \\ Information Technology, Visakhapatnam, AP, India \\ ${ }^{2}$ Dept. of Department of Computer Science and Engineering, Pydah College of \\ Engineering and Technology, Visakhapatnam, AP, India \\ ${ }^{1}$ mallikblue@gmail.com, ${ }^{2}$ neerajasreerama@gmail.com
}

\begin{abstract}
MANET is a truncation for a special versatile appointed system. It is additionally alluded to as a remote impromptu system and it is a continuous self-designing, framework-free system of phones that are associated with no utilizing wires. In MANET engineering, gadgets can proceed separately in the direction of any path and in this way, change their connections to various gadgets now and then. Since MANETs are transportable, they use remote associations with interface with various systems. In this article, a diagram of MANET alongside its use in remote frameworks will be discussed. A short thought in regards to the kinds of MANET designs and their points of interest and inconveniences in remote correspondence systems is depicted in this paper.
\end{abstract}

Keywords: Characteristics of MANET, Architecture, Routing conventions, Challenges and applications of MANET

\section{Introduction}

The recent years have seen a fast heightening in the sphere of portable figuring due to the multiplication of reasonable and generally accessible remote gadgets. In this manner, it has opened an immense open door for scientists to take a shot at Ad Hoc Networks. In a MANET, the hubs inside each other's remote transmission range can convey straightforwardly; in any case, hubs outside each other's range need to depend on some different hubs to handoff messages.

In this manner, a multi-bounce situation happens, where the bundles sent by the source host need to be transferred to influence them to achieve the goal hub. MANET is something that meets its expectations, without any assistance from the current foundation or any sort of settled stations.

This announcement can be formally defined by characterizing a specially appointed system as a self-governing arrangement of versatile hosts (MHs), (additionally filling in as switches) which can be associated with the help of remote connections, whose association frames a correspondence that is organized to be displayed as a subjective correspondence chart.

This is as opposed to the outstanding single bounce cell arrangement that demonstrates the backings of the requirements of remote correspondence with the introduction of Base Stations (BSs) as passages. In these cell systems, correspondences between two portable hubs depend

Article history:

Received (April 1, 2019), Review Result (May 5, 2019), Accepted (June 6, 2019) 
on the wired spine and the settled (BSs). In the MANET, such a foundation doesn't exist and the system topology may powerfully change capriciously because hubs can be allowed to move. Concerning the method of activity, specially appointed systems are fundamentally peer-topper multi-jump portable remote systems where data parcels are transmitted in a "store-and-forward" way from a source to a self-assertive goal, through middle hubs.

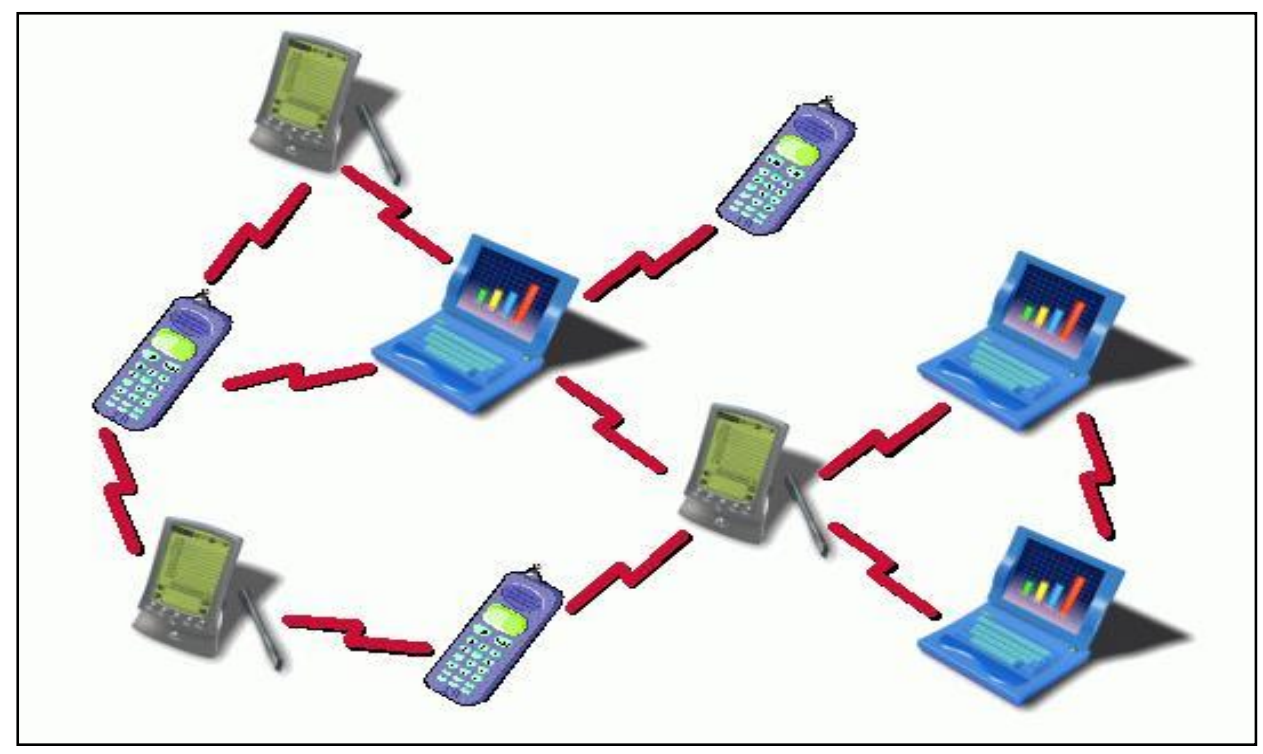

Figure 1. MANET

\section{Features of MANET}

Independent Terminal Nodes in MANET can serve the capacity of the host and additionally perform works as switch [2][3].

Appropriated operation: As there exists no focal control framework out of sight, the control and administration activity of systems are appropriated among the terminals. All hubs are teamed up and work themselves and every hub goes about as required.

Multi-bounce Routing: Single bounce and multi-jump are the fundamental sorts of specially appointed steering calculations. If we discuss the structure and usage, at that point, a singlejump MANET could be more straightforward than a multi-bounce. Conveying bundles in a multi-bounce are transmitted through the excess of one hub to the goal.

Dynamic Network Topology: The Network topology may change quickly and eccentrically as hubs are portable. Hubs that exist in each other's range can confer clearly and are responsible for dynamically finding each other.

Self-Creation, Self-Organization, and Self Administration: Portable Ad hoc Network can be quickly sent as there is no requirement for the point by point plane of establishment or wiring.

Self-coordinated terminal: Every portable hub in the impromptu systems acts like both a host and a switch. It implies that the hubs are sending the bundle to furthermore forward the parcel or course the parcel.

The flexibility of connection limit: The compact exceptionally named frameworks each one of the three sorts; controlling, for instance, unicast, multicast, and impart are used. When the source center point is required to send a package to another center point that has no speedy relationship with the source center. Then source center point conveys the RREQ message to the MANET structures. When the center point gets the course request for a message, it develops 
against the pivot route to the source and objective to re-convey the package. When the package comes to an objective center point than an objective center unicast, the course answers the message to the source.

\section{MANETs architectural model}

The MANETs compositional model safeguards the honesty of the IP engineering by considering the particularities of MANETs.

MANET (Mobile Ad hoc Network) has alluded to a multi jump parcel-based remote system, an arrangement of versatile hubs, that can convey and move, with no utilization of any sort of settled wired framework. These are self-arranging and versatile systems that can be shaped and distorted with no need for any incorporated organization. This restrictive trademark permits the utilization of MANETs in numerous specific regular citizen and military circumstances and also in the developing innovation of sensor systems [2][3].

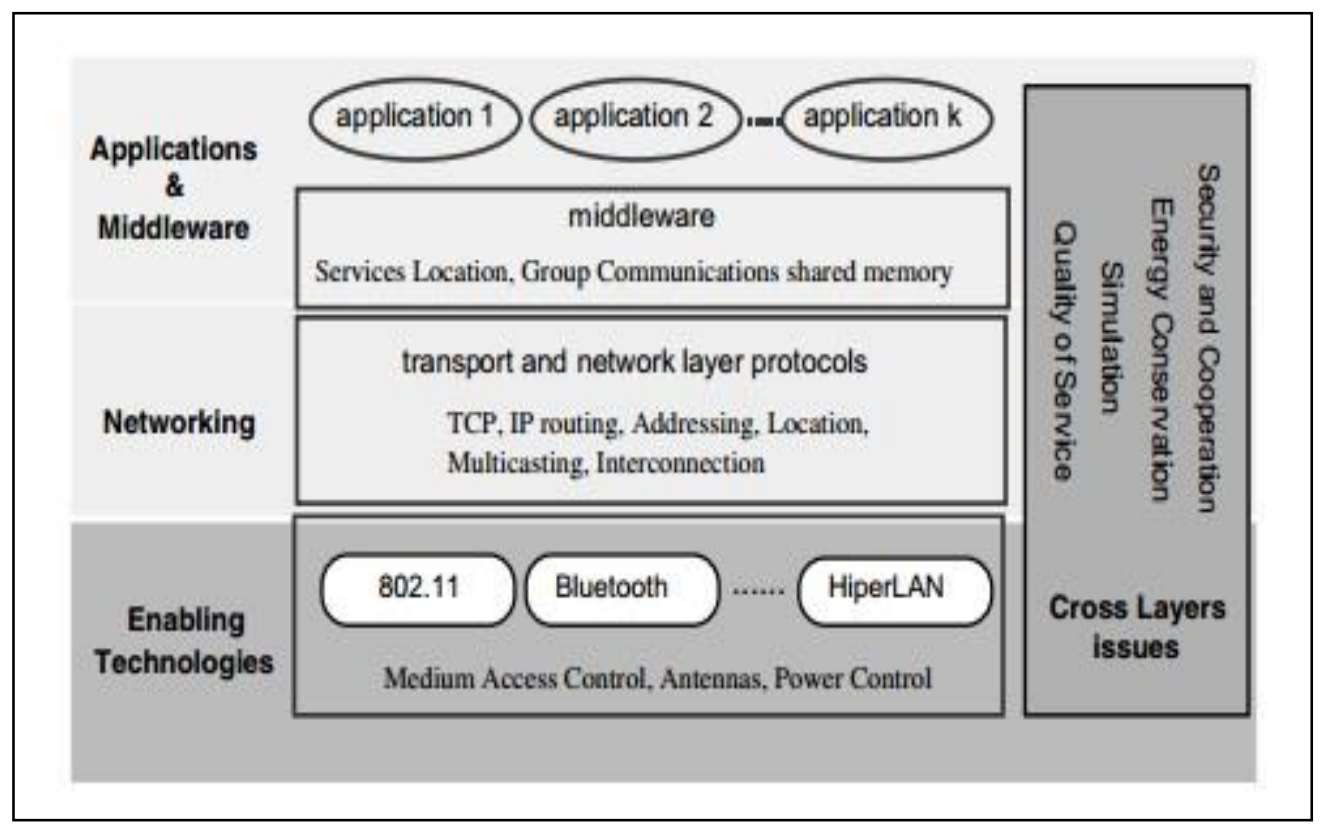

Figure 2. Architecture of MANET

The engineering of MANET from the [figure 2]. given above can be isolated into 3 primary parts and are as follows

(1) Empowering Technologies

(2) Systems administration

(3) Middleware and Application

The above 3 fundamental characterizations of MANET can be discussed as per the following:

\subsection{Empowering technologies}

Taking into consideration of the scope territory, it is additionally partitioned into different classes as:

Boycott (Body Area Network): The correspondence run is 1-2 meters and hence BAN gives the availability to gadgets that might be appended to wearable PCs. 
Container (Personal Area Network): The correspondence run is till 10 meters and hence PAN associates the cell phones to the other cell phones or stationary gadgets.

WLANs (Wireless Local Area Networks): The correspondence run is 100-500 meters for a singular building or gathering of structures. WAN (Wide Area Network) and MAN (Metropolitan Area Network) are versatile multi-bounce remote systems that go through different difficulties like security, tending to area administration, and so forth.

\subsection{Systems administration}

In MANET, almost all of the guideline functions of the Networking conventions need to be upgraded for the self-arranging, dynamic, unsteady and shared correspondence condition. The most important focal point of the administration of the system conventions is to utilize the 1 jump transmission administrations that are acquired by the empowering advancements to make end-to-end solid administrations, from a sender to 1 receiver. For building a closure to end correspondence, the sender should first uncover the recipient present in the network. The principal reason for a sector benefit is to strictly separate the address of the beneficiary gadget to the present sector of the network.

\subsection{Middleware and applications}

The latest enhanced technologies like Wi-Fi, Bluetooth, IEEE 802.11, WiMAX, and Hyper LAN massively facilitate the inclusion of impromptu revolution and the latest special appointed systems administration applications primarily in certain spheres like disaster administrations, disaster recovery, and condition observing. The pliability of MANET is appealing for few functional situations, e.g. in PAN, home systems administration, law authorization tasks, business and instructive applications, and sensor organizer. There are specifically appointed structures that receive the philosophy of not containing a middleware, but do depend upon each application to deal with each one of the administrations it needs.

\section{Kinds of MANET}

Mobile Ad-Hoc Networks covers countless viewpoints for giving its administrations. This can be accomplished by utilizing the kinds of MANET according to the necessities at the purpose of circumstances. There are three noteworthy sorts of MANET which are generally utilized [3]:

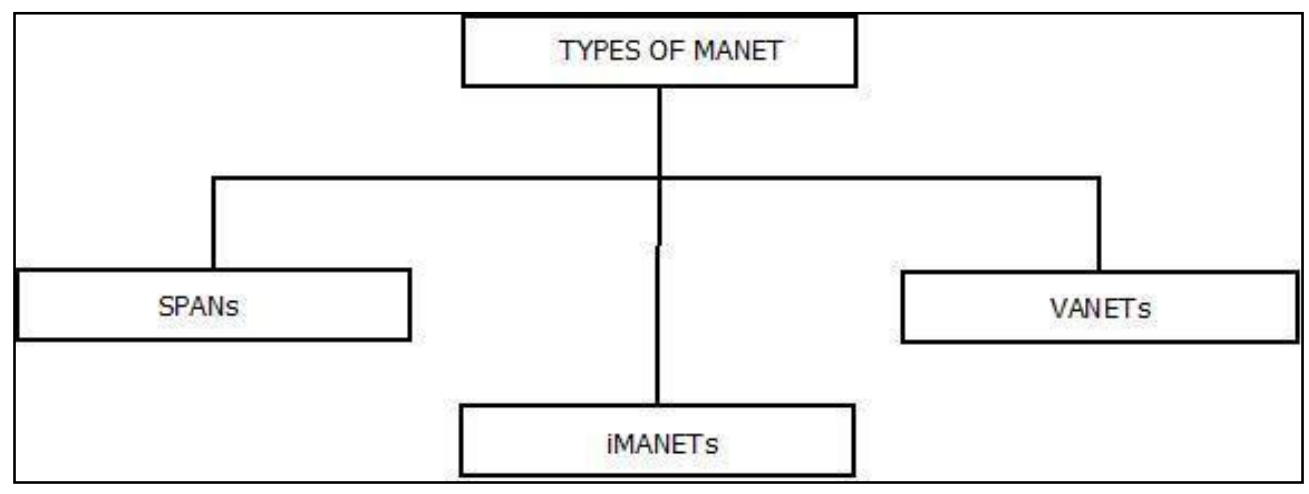

Figure 3. Types of MANET 


\section{A. SPANs}

SPANs remain for Smartphone-Based Mobile Ad-Hoc Networks. The kind of MANET that can be utilized crosswise over cell phone gadgets by making a shared system through the assistance of Wi-Fi and Bluetooth Technology.

B. IMANETs

IMANETs remain for Internet-Based Mobile Ad-Hoc Networks. The kind of MANET chips away at the idea of systems administration conventions including User Datagram convention, Transmission Control Protocol, Internet convention, and Routing data convention.

C. VANETs

VANETs remain for Vehicular Mobile Ad-Hoc Networks. The kind of MANETs which can be utilized crosswise over vehicles and street wellbeing gadgets for correspondence reason which thus is in charge of smooth and secure vehicle conduct over the streets.

\section{MANETs categories}

MANETs can be extensively characterized into two sorts:

A. SINGLE HOP

Single bounce MANETs are those which contain the greatest separation of one jump from another jump. Bounce implies a hub or a gadget or a switch. This implies a distributed system that can be called a solitary bounce MANET. For example- Bluetooth Network.

\section{B. MULTIHOP}

Multihop MANETs are those in which at least more two gadgets can be put a jump separated from each other. For example- Police Network.

\section{Directing protocol in manet}

There exist some unique and specific steering conventions that exist in MANETs. Steering conventions can be classified into 3 types: Proactive, Reactive Directing, and Hybrid conventions. The directing conventions are polished to deal with a large number of hubs having limited assets. The significant stress in steering convention is exhibiting the hubs in different spots. It is essential to reduce the directing message overhead notwithstanding the growing number of portable hubs [1]. One more essential issue is to keep up the measure of directing table as negligible on the grounds, so in the span of steering convention is larger than it, it can affect the control bundle exchanged within the system.

Steering convention is grouped on how and at what time the course is found, though anyway both choose the most limited path to the goal.

(1) Proactive routing protocols

This type of steering convention uses state directing calculations which gushes the interface data about its neighbors most of the time. Proactive directing convention can store the steering data and keeps pace with the data model by dealing with the control bundle from their neighbors. The occurrences of these are DSDV, OLSR, WRP, etc.

(2) Receptive routing protocols

Receptive steering conventions decrease overheads which are present in proactive conventions. This uses remove vector directing calculation and creates the course to the given destination when a hub asks for it by starting the course revelation process. Numerous responsive directing conventions are present that are attainable in MANET like DSR, AODV, TORA, LMR, etc [4][5][6][7].

(3) Crossbreed routing protocols 
This is the mix of responsive and proactive directing conventions. The case Hybrid steering conventions are ZRP, BGP, EIGRP. The following demonstrates distinction among the Proactive, Reactive steering, and Hybrid conventions in different highlights [8].

Table 1. Comparison between conventions

\begin{tabular}{|c|c|c|}
\hline Parameters & AODV & DSR \\
\hline Scalability & No & No \\
\hline Reliability & Yes & Yes \\
\hline Throughput & $\begin{array}{l}\text { Poor for more than } 20 \text { mobile } \\
\text { nodes }\end{array}$ & $\begin{array}{c}\text { Decrease as mobility } \\
\text { increases }\end{array}$ \\
\hline Load Balancing & No & No \\
\hline Congestion Control & No & No \\
\hline Overall Complexity & Medium & Medium \\
\hline Routing Philosophy & Flat & Flat \\
\hline Routes Maintained & Route Table & Route Cache \\
\hline Loop Free & Yes & Yes \\
\hline Multiple Route Support & No & Yes \\
\hline Overhead & Low & Medium \\
\hline Protocol Type & Distance Routing & Source Routing \\
\hline Route Reconfiguration & Erase Route; Notify Source & Erase Route; Notify Source \\
\hline $\begin{array}{l}\text { Expiry of Routing } \\
\text { Information }\end{array}$ & Yes & No \\
\hline
\end{tabular}

\section{Utilizations of manet}

A few unmistakable MANET applications are as follows [2][3]:

(1) Military field

Specially appointed systems administration can allow armed force to abuse profit of traditional system aptitude for protecting any data arrangement among vehicles, military, and home office of data.

(2) Helpful work

To encourage the business settings, the need for deliberate processing is extremely noteworthy outside to office air and surroundings when contrasted with the internal condition. Individuals require getting the outside gatherings for trading the data in addition to coordinating with each other in regards to any doled out errand.

(3) limited level

Specially appointed systems can openly connect with quick, moreover transitory hypermedia organized by methods for smartphones imparting the information to every one of the candidates, for example- classroom and gathering. Extra legitimate and restricted level demand might be in a local system whose gadgets are capable of interconnecting to straight in trade the data.

(4) Skillet and Bluetooth

A PAN is restricted and a modest scope arrangement where these gadgets for the most part have a place with a predetermined individual. Restricted range MANET, for example, Bluetooth can make easier the trade between a few versatile gadgets like PCs, and phones. 
(5) Business sector

A specially appointed network can be used for reducing the calamity for the misfortune of help battles; for example, in the surge, fire, or earthquake. These calamity-saving techniques should take place where harmed and non-existing transmissions structure and fast arrangements of a transmission organization are needed.

(6) Sensor networks

Supervising home machines with MANETs for both the cases like close-by and remotely for the following items like animals and climate detection-related exercises.

(7) Reinforcement services

These include the freedom activities, disaster recovery, analysis/status or record delivering healing facilities, and substitution of the stationary framework.

(8) Instructive part

The correspondence offices for PC are generated gathering the rooms or classrooms or research centers.

\section{Conclusion}

A gigantic progression has been seen in the field of versatile correspondence in a previous couple of years. Therefore different open doors are opened up in the field of specially appointed systems nowadays. MANET is a gathering of remote portable hosts which constructs an impermanent system without the necessity of any brought together organization or spine bolster administrations. MANET substantiated itself as an adaptable system these days. However, it is very untrustworthy because of its less assault dealing ability that means it has little resistance to assaults. Directing is the most extreme piece of any system which likewise holds its centrality in MANET engineering. Different sorts of directing conventions are utilized for various kinds of MANET designs to guarantee the coveted way for transmission of message parcels amongst source and goal.

\section{References}

[1] Yick, Jennifier, Bishwanth Mukherjee, and Dipak Ghosal, "Wireless sensor network survey," Computer Networks, vol.52, no.12, 22 August, pp.2292-2330, (2008)

[2] Alex Hinds, Michael Ngulube, Shaoying Zhu, and Hussain Al-Aqrabi, "A review of routing protocols for mobile ad hoc networks," International Journal of Information and Education Technology, vol.3, no.1, Feb, pp.1-5, (2013)

[3] Mr. L Raja, Capt. And Dr. S Santhosh Baboo, "An overview of MANET: applications, attacks and challenges" International Journal of Computer Science and Mobile Computing, vol.3. no.1, Jan, pp.408-417, (2014)

[4] Kayna Mahajan and Harjit Singh, "MANET its types, challenges goals and approaches: A review," International Journal of Science and Research, vol.5, no.5, May, pp.1591-1594, (2016)

[5] Singh G. and Singh A. "Performance evaluation of AODV and DSR routing protocols for VBR traffic for 150 nodes in MANETs," International Journal of Computational Engineering Research (ijceronline. com), vol.2, pp.1583-1587, (2012)

[6] Kaur R. and Rai M.K., "A novel review on routing protocols in MANETs," Undergraduate Academic Research Journal (UARJ), vol.1, pp.103-108, (2012)

[7] Dhenakaran S.S. and Parvathavarthini A. "An overview of routing protocols in mobile ad-hoc network," International Journal of Advanced Research in Computer Science and Software Engineering, vol.3, pp.251-259, (2013)

[8] Priya Dharshini. R, Prabhu. V, and Rajes Singh.S, "Research on implementation and comparison of routing protocols in MANET Using NS2,” International Journal of Science and Research, vol.3, no.4, April, (2014) 
Comparison of Routing Convention in MANET: A Survey

[9] Kanchan Ubnare, Prof. Navneet Manghi, and Prof. Vimal Shukla, "A comparative study of routing protocols in mobile Ad-Hoc networks," International Journal of Emerging Technology and Advanced Engineering, vol.4, no.3, March. pp.833-837 (2014) 